\title{
Preface: Special topic on photocatalytic or electrocatalytic reduction of $\mathrm{CO}_{2}$
}

\author{
Yi Xie \\ Hefei National Laboratory for Physical Sciences at the Microscale, CAS Center for Excellence in Nanoscience, iCHEM, University of Science \\ and Technology of China, Hefei 230026, China
}

Received October 15, 2020; accepted October 15, 2020; published online October 21, 2020 $\begin{array}{ll}\text { Citation: } & \text { Xie Y. Preface: Special topic on photocatalytic or electrocatalytic reduction of } \mathrm{CO}_{2} \text {. Sci China Chem, 2020, 63: 1703-1704, https://doi.org/10.1007/ } \\ \text { s11426-020-9892-8 }\end{array}$

$\mathrm{CO}_{2}$ reduction technology, which converts greenhouse gases into industrial feedstock or liquid fuels using renewable electricity or solar energy, is considered a promising energy conversion technology. The reaction uses $\mathrm{CO}_{2}$ molecules as the raw material, which can effectively reduce the atmospheric $\mathrm{CO}_{2}$ content, thereby curbing the greenhouse effect. Moreover, by designing rational catalysts, $\mathrm{CO}_{2}$ molecules can be directly converted into usable fuels, which effectively alleviate the global energy crisis. With the deterioration of the ecological environment and increase in energy demand, $\mathrm{CO}_{2}$ reduction technology has received increasing attention and has become an important research area in the fields of chemistry and energy as well as in the chemical industry.

Although renewable electricity or solar energy-driven $\mathrm{CO}_{2}$ reduction to fuels has great potential, the reduction of chemically inert $\mathrm{CO}_{2}$ molecule is very challenging and complex owing to the high $\mathrm{C}=\mathrm{O}$ dissociation energy of $750 \mathrm{~kJ} \mathrm{~mol}^{-1}$ and similar reduction potential of various reduction products. Hence, it is highly crucial to develop innovative strategies for modulating the atomic and electronic structures of photo/ electrocatalysts, with efforts to tailor the reaction energy barrier and/or change the reaction path, thus yielding boosted reaction activity and product selectivity.

Recently, significant progress has been achieved in the research of catalysts. Many emerging catalysts, such as

*Corresponding author (email: yxie@ustc.edu.cn) hollow nanostructures, single-atom catalysts, and molecular catalysts, are widely used in $\mathrm{CO}_{2}$ reduction and have yielded good results. Meanwhile, numerous novel devices, such as anion exchange membranes and flow cell electrocatalytic systems, have also been proposed to improve the $\mathrm{CO}_{2}$ conversion efficiency. Furthermore, China has invested large amounts of funds to support the development of $\mathrm{CO}_{2}$ reduction technology. Consequently, many researchers have dedicated themselves to this research and have developed several potential catalytic systems.

In this special topic, five research articles and communications are presented, providing a glimpse of the current progress in the $\mathrm{CO}_{2}$ photo/electrocatalytic reduction. Some novel catalysts, such as $\mathrm{CuInS}_{2}$ hollow nanostructures, Co single atoms, $\mathrm{Au}$ nanosheets, and molecular $\mathrm{Ni}$ catalysts, have been designed to promote the performance of $\mathrm{CO}_{2}$ photo/electrocatalytic reduction. Additionally, density functional theory calculations have been performed to reveal the reaction mechanism. However, this collection comprises a limited selection of new research results. We only aim at illustrating some recent developments in $\mathrm{CO}_{2}$ reduction.

Despite its great importance, $\mathrm{CO}_{2}$ reduction still suffers low conversion efficiency and poor product selectivity. Moreover, current research is mainly focused on $\mathrm{C} 1$ products, while the fabrication of value-added $\mathrm{C} 2$ products with high energy density is more promising. Therefore, to achieve efficient and selective $\mathrm{CO}_{2}$ reduction performance, the development of active catalytic systems is highly desired. We 
hope that this special topic will help researchers achieve an improved understanding of $\mathrm{CO}_{2}$ photo/electrocatalytic re- duction and guide the design of enhanced high-performance photo/electrocatalytic systems.

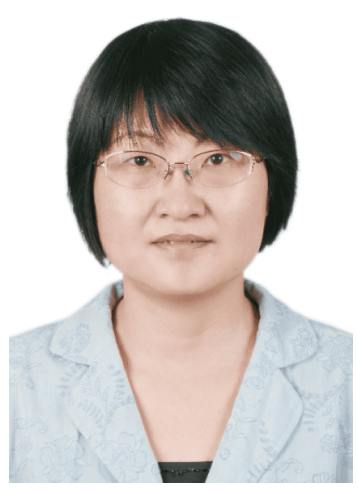

Professor XIE Yi received her B.Sc. from Xiamen University (1988) and her PhD from the University of Science and Technology, China (USTC, 1996). In May 1996, she joined the Department of Chemistry, USTC as a faculty member. She is now a Principal Investigator at the Hefei National Laboratory for Physical Sciences at the Microscale and a full Professor at the Department of Chemistry, USTC. She has also received several awards, including the L'Oréal-UNESCO for Women in Science International Award, The World Academy of Sciences (TWAS) Prize for Chemistry, the IUPAC Distinguished Women in Chemistry/Chemical Engineering, and the Nano Research Award. She was elected as a member of the Chinese Academy of Sciences in 2013 and a TWAS member in 2015. Her current research focus is the design and synthesis of inorganic functional solids in an effort to shape their electronic and phonon structures. 